Review Article

\title{
Syndrome Differentiation of Diabetes by the Traditional Chinese Medicine according to Evidence-Based Medicine and Expert Consensus Opinion
}

\author{
Jing Guo, Hongdong Chen, Jun Song, Jia Wang, Linhua Zhao, and Xiaolin Tong \\ Guanganmen Hospital, China Academy of Chinese Medical Sciences, Beijing 100053, China \\ Correspondence should be addressed to Linhua Zhao; melonzhao@163.com and Xiaolin Tong; tongxiaolin66@126.com
}

Received 10 April 2014; Accepted 2 July 2014; Published 14 July 2014

Academic Editor: Zhao-Xiang Bian

Copyright (c) 2014 Jing Guo et al. This is an open access article distributed under the Creative Commons Attribution License, which permits unrestricted use, distribution, and reproduction in any medium, provided the original work is properly cited.

In Chinese medicine, diabetes belongs to the category of "Xiaoke disease (disease with symptoms of frequent drinking and urination)"; in the traditional sense, its pathogenesis is "Yin deficiency and dryness-heat." However, over time, changes in the social environment and lifestyle have also changed the use of traditional Chinese medicine (TCM) in diabetes. In this study, we performed diabetes syndrome differentiation using TCM according to evidence-based medicine and expert consensus opinion.

\section{Introduction}

Diabetes mellitus (DM) is often caused by the consumption of a high fat and calorie diet. It has a high prevalence and can often lead to complications that seriously affect the quality of life of sufferers. In 2013, according to the latest statistics of the International Diabetes Federation (IDF), the global prevalence of diabetes among adults aged $20-79$ was $8.3 \%$. The total number of patients with diabetes worldwide was estimated to be 382 million, which was predicted to rise to nearly 592 million by 2035 . Therefore, research on the prevention and treatment of diabetes is critical and represents a great challenge for the medical profession.

In recent years, Chinese medicine has made great progress toward the prevention and the treatment of diabetes, and its curative effects have been widely recognized. The clinical effects of the kai-yu-jiang-zhuo decoction [1] are the same as metformin; therefore, the kai-yu-jiang-zhuo decoction could be recommended clinically. In one study [2] assessing the ability of the Chinese herbal medicine Tianqi to reduce progression from impaired glucose tolerance to diabetes, there was a significant difference in the number of subjects who had normal glucose tolerance at the end of the study between the Tianqi (63.13\%) and placebo groups, respectively $(46.60 \%)$. Cox's proportional hazards model analysis demonstrated that Tianqi reduced the risk of diabetes by $32.1 \%$ compared with placebo. Similarly, tang-min-ling pills [3] could effectively reduce glycosylated hemoglobin levels and fasting blood glucose (FBG) and improve islet $\beta$ cell function. As the main ingredient in Coptis, Berberine $[4,5]$ also has good hypoglycemic effects and improves insulin resistance.

Treatment based on syndrome differentiation is the basic principle of illness and treatment in traditional Chinese medicine (TCM). To improve symptoms, individualized treatment plans are more scientific and superior. A previous study [6] suggested that the Chinese medicinal dialectical treatment of patients with diabetes was highly effective. In addition, dialectical treatment is relatively flexible, causes less adverse reactions, and is safe and reliable. These characteristics can effectively improve the quality of life of diabetic patients and are worth assessing clinically. However, due to the inadequate international understanding of the culture of TCM, syndrome differentiation is often avoided or disregarded, which reduces the potential benefits of TCM.

In TCM [7], the diagnoses and treatment of Xiaoke disease were traditionally based on "three excessive and one loss" symptoms, excessive fluid drinking, excessive foodconsumption, excessive urination, and weight loss and its core pathogenesis is "Yin deficiency and dryness-heat." However, $80 \%$ of type 2 diabetes patients do not have these three 
typical symptoms, so are very different form Xiaoke. Approximately $85 \%$ of type 2 diabetes patients are overweight or obese, suggesting that these obese diabetes patients form the majority of the diabetic population. In addition, compared with prior living environments, the modern diet has changed significantly, which has resulted in a significant increase in the diabetic population. Fewer individuals are thin, and an increasing number of people are obese. Thus, physicians gradually realized that the typical SanXiao symptoms (three types of diabetes that, resp., involving the upper-jiao, middlejiao, and the lower warmer) usually develop later during the pathogenesis of diabetes, and so most people with diabetes do not present with these symptoms. The traditional TCM theory of Yin deficiency and dryness-heat is more difficult to obtain a satisfactory curative effect during the treatment of diabetes; therefore, novel theories have been proposed. Diabetes symptoms are complex, and physicians do not have unified opinions regarding the pattern identification based the syndrome differentiation of diabetes. Guidelines of Prevention and Treatment of Diabetes by TCM, the first guidelines for diabetes, which was issued in 2007 as a project funded by the State Administration of Traditional Chinese Medicine, promoted the diagnosis and treatment of diabetes and its complications. The guidelines were proposed and revised repeatedly by members of the standing committee of diabetes branch of the China Association of Chinese medicine and were confirmed by the domestic renowned diabetes experts Linlan, Zhang Farong, Li DeLin, and Cheng Yichun. With the unification of TCM and Western medicine terminologies related to diabetes and its complications, diabetes-related terminology gradually became normalized and standardized. In this study, we perform diabetes syndrome differentiation of TCM based on the guidelines (2007) and evidence-based medicine.

\section{Syndrome Differentiation}

"TCM syndrome differentiation and evaluation standard of DM", "Guidelines of Prevention and Treatment of Diabetes by TCM, 2007," and "DM treatment using integrated traditional Chinese and Western medicinal therapy" have all been proposed for the TCM-mediated differentiation of the clinical stages of DM [8, 9]. In one study [10], stagnancy, heat, deficiency, and damage were thought to be the four stages of diabetes, and collateral damage existed through the course of the disease even before the diagnosis. The collateral damage in different degrees could be defined as "collateral qi obstruction, collateral qi stagnation, collateral blockage, and collateral damage." The study also suggested that the TCM-mediated differentiation with the clinical stages of $\mathrm{DM}$ is indispensable. Therefore, we performed syndrome differentiation based on the clinical stages of diabetes.

\subsection{Syndrome Differentiation of DM}

2.1.1. QI Stagnation due to Liver Depression. According to modern medical research [11], patients with diabetes often exhibit aggravated emotional tension, which is consistent with the theory of TCM that negative emotions could lead to diseases. Liver depression could lead to qi stagnation and result in some emotional symptoms. This was the first stage of diabetes, and the characteristic was stagnancy. Therefore, soothing the liver and adjusting qi are the main therapeutic principles. This type of diabetic patients shows some emotional symptoms such as depressed mood, like frequent sighing, nervousness, distention, and fullness in the chest and rib-side. The patients usually have a pale tongue with thin white moss and a stringy pulse. Modified Xiao Chaihu decoction, a classic Chinese ancient prescription, was commonly used to treat this type of diabetic patients; some herbs like Bupleurum, Scutellaria baicalensis, Pinellia ternata, and Ginseng were included in this decoction [12-15].

2.1.2. Liver and Stomach Heat Stagnation. A symptom analysis of 2518 obese patients with type 2 diabetes [16] demonstrated that there were 1332 cases of liver and stomach heat stagnation syndrome, accounting for $52.9 \%$ of all the cases, suggesting that it was an important type in diabetes syndrome differentiation. Liver and stomach heat stagnation belong to the stagnancy and heat stages of diabetes. The patients of this type showed some emotional and digestive symptoms such as irritability, distention and fullness in the chest and rib-side, drinking too much fluids and the production of increased urine, eating too much food, hunger, experiencing a bitter taste, dry mouth, and constipation. And patients usually have a red tongue, and a rapid and stringy pulse. Clearing stagnation-heat of liver and stomach is an important therapeutic principle for this type. A modified Da Chaihu decoction, one of classic Chinese ancient prescriptions recorded in Treatise on Cold Pathogenic and Miscellaneous Diseases, was used to treat such diabetic patients. Some Chinese herbs like Bupleurum, Chinese rhubarb, Scutellaria baicalensis, Citrus aurantium, Radix Paeoniae Rubra, and so forth were included in this formula $[17,18]$.

2.1.3. Phlegm and Heat Stasis. A study by Gan et al. [19] showed that phlegm and heat stasis syndrome was a common type in the early and middle stages of diabetes and accounted for more ratios particularly in patients who smoked and drank alcohol. Zhou et al. [20] investigated 344 patients with type 2 diabetes and found that 101 cases (29.4\%) belonged to phlegm and heat stasis syndrome. This syndrome often appears in the "heat" stage of diabetes, and the patients are relatively obese because in the theory of Chinese medicine, "obese people tend to have copious phlegm." Patients with this type may have some symptoms such as abdominal obesity, a sense of chest suppression, abdominal distention, and dry mouth. They might also prefer cold drinks, drink much more fluids, and be irritable and have a bitter taste in their mouth as well as constipation. Patients also have a red and fat tongue with yellowish greasy moss, yellow urine, and a stringy and smooth pulse. Reducing heat and removing phlegm is the therapeutic principle in this syndrome, and a modified Xiao Xianxiong decoction, a classic Chinese ancient prescription, is used to treat such diabetic patients. Some Chinese herbs 
like rhizoma coptidis, Pinellia ternata, snakegourd seed, and so forth are included in this formula [21-24].

2.1.4. Excess Heat in the Stomach and Intestine. Both Tong and Wang's studies $[16,25]$ demonstrated that "excess heat in the Stomach and intestine" was one of main syndromes of diabetes. This syndrome generally occurs in the diabetic middle stage or in the "heat" stage. In the middle stage of diabetes, patients eat large amounts of food, which stagnate and form heat in the stomach and intestine. As such, its principal symptoms are abdominal fullness and distention, constipation, a bitter taste and dry mouth, halitosis, thirst with a desire for cool water, drinking and eating too much, and hunger. Patients usually have a red tongue with yellow moss and a rapid strong pulse. To remove the heat, a modified Dahuang Huanglian Xiexin Decoction is regarded as the main prescription which includes Chinese rhubarb and rhizoma coptidis and so forth [25-27].

2.1.5. Intestinal Damp and Heat Syndromes. According to the findings of Zhao et al. [28] in a study using classical prescriptions to treat different diseases, the Gegen Qinlian decoction could be used to treat type 2 diabetes with concurrent intestinal damp and heat syndromes, with a good clinical efficacy. An additional study [29] showed that Gegen Qinlian decoction could significantly improve the intestinal damp and heat syndrome scores of patients with type 2 diabetes and could effectively control blood glucose with a success rate of $88.6 \%$. Another study [30] revealed that the morbidity of type 2 diabetes with damp and heat syndrome was $30.7 \%$ and that the location of disease was in Fu-organs. In addition, this syndrome has unique features. The intestinal damp and heat syndromes always appear in the diabetic middle stage or during the heat stage. Its principal symptoms are thirst with no desire to drink, hunger with no desire to eat, a bitter taste, a sticky and greasy sensation in the mouth, and abdominal distention. Patients also show a red tongue with yellow and greasy moss and a slippery pulse. When damp and heat affect the large intestine, smelly greasy stools might also form. To reinforce the spleen and stomach and remove the heat and dampness, a modified Gegen Qinlian decoction, one of classic Chinese ancient prescriptions, is used to treat such diabetic patients. Some Chinese herbs like kudzuvine root, Scutellaria baicalensis, rhizoma coptidis, and so forth are included in this formula $[29,31]$.

\subsubsection{Deficiency of Body Liquid due to Excessive Heat Syn-} drome. A study by Gan and Chen [32] suggested that excessive heat injuring liquid syndrome was a principle syndrome of diabetes. Consistent with this, Zhang et al. [33] reached the same conclusion after investigating 1490 cases of type 2 diabetes using clinical syndrome differentiation. The deficiency of body liquids due to excessive heat syndrome is more commonly found in the diabetic middle-late stage or the heat and deficiency stages. Impacted by the fire and heat pathogens from the early and middle stages of diabetes, qi is consumed and liquids are injured gradually. As such, its principle symptoms are dry throat and mouth, thirst with a desire for cool water, overeating and hunger, frequent micturition volume, irritability, bitter taste, red urine, and constipation. Patients also commonly have a red tongue with yellow fur and a rapid pulse. To sooth the heat and promote fluid production, a modified Xiaoke Wan or Baihu Tang, belonging to classic Chinese ancient prescriptions recorded in Treatise on Cold Pathogenic and Miscellaneous Diseases, is used to treat such diabetic patients. Some Chinese herbs like Gypsum, Rhizoma Anemarrhenae, Liquiritia Glycyrrhiza, and so forth are included in this formula [34-36].

2.1.7. Dual Deficiency of Qi and Yin. Based on her 40 years of clinical experience, Lin and $\mathrm{Ni}$ [37] proposed a theory called III-type differentiation, which proposes that the dual deficiency of qi and yin syndrome was one of basic syndromes of diabetes. Many other professors, including Xu et al. [38], Mao et al. [39], and Li et al. [40] also concluded that the dual deficiency of qi and yin syndrome was a common syndrome of diabetes. The dual deficiency of qi and yin syndrome occurs in the late diabetic or the deficiency stage. The fire and heat pathogens further dissipate the primordial qi of zang-fu organs, and then the generalized qi is consumed. In addition, fire and heat pathogens scorch liquids and damage yin. Therefore, the main symptoms are dry throat and mouth, thirst with a large intake of fluid, fatigued spirit and lack of strength, shortness of breath and reluctance to speak, emaciation of the body, limp aching lumbus and knees, spontaneous and night sweats, feeling palm and arch fever, upset, palpitations, insomnia, a red tongue with scant liquids and thin white dry tongue fur, and a fine rapid pulse. Boosting qi and nourish yin is one of the important therapeutic principle in this type, and a modified Shengmai Yin decoction, a classic Chinese ancient prescription, is used to treat such diabetic patients. Some Chinese herbs like, Ophiopogon japonicas, Schisandra chinensis, ginseng, and so forth are included in this formula [41, 42].

2.2. The Stage of Diabetes Complications. During the diabetic complications stages, treatment requires the combination of disease and syndrome differentiation due to its complexity. In general, the deficiency is increasingly aggravating, and so qi-blood-liquid deficiency and the function of internal organs decline. It belongs to late stage of diabetes; liver and kidney insufficiency and deficiency in both yin and yang are its endpoints. The main syndromes in this stage include insufficiency of the liver and kidney and detrimental yin and yang $[10,15]$.

2.2.1. Insufficiency of the Liver and Kidney Syndrome. The main symptoms are urinary frequency, turbid unctuous, and limp aching and lumbus and knees, which are accompanied by additional symptoms including blurred vision, dizziness, tinnitus, red tongue with some fur, and a fine rapid pulse. Modified Qiju Dihuang Wan, which includes the fruit of Chinese wolfberry, chrysanthemum, Chinese yam, and Cornus officinalis, is used to treat these diabetic patients by enriching the liver and kidney with essence and increasing blood supply [43-45]. 
2.2.2. Dual Deficiency of Yin and Yang Syndrome. Patients with this syndrome exhibit symptoms including urinary and nocturia frequency, which can be accompanied by feeling palms and arches fever, being upset, dry throat or mouth, limp aching lumbus and knees, fear of the cold, icy cold limbs, and a forceless fine sunken pulse. Enriching yin and supplying yang is an important therapeutic principle; modified Jingui Shenqi decoction, which includes the Chinese herbs adhesive rehmannia dried root, Chinese yam, Fructus Corni, cassia twig, and monkshood, is commonly used to treat these diabetic patients [46-49].

Studies [50] have shown that the characteristic pathophysiological mechanism of chronic diabetic complications is root deficiency and tip excess. Deficiency and static blood occur throughout several complications. The dual deficiency of qi and yin, phlegm turbidity, and static blood obstructing the network vessels form the common pathological basis of diabetic chronic complications. Studies [51] using the collateral disease theory have determined that static blood obstructing the collateries is the pathological basis for diabetic microangiopathy; therefore, treatment should be aimed to promote blood circulation and remove obstruction in vessels throughout the whole process. A large number of clinical observations and scientific studies have confirmed that capillaries can be protected and diabetic microvascular complications prevented and treated using drugs that accelerate blood flow during early-mid diabetes.

Generally, treatments for diabetic complications should target phlegm, static blood, and additional pathological factors, except for insufficiency of the liver and kidney and dual deficiency of qi and yin, for more comprehensive and thorough evidence-based medicine.

\section{Diabetic Patterns and Correlation Indices}

3.1. Pattern Types according to the Function of Insulin. Studies [52-54] have demonstrated that diabetic syndrome differentiation is closely correlated with insulin function, which provides an objective basis for syndrome differentiation using TCM. During the pathogenesis of diabetes, islet $\beta$-cell function changes from the compensatory period to the mildly decompensated period, severe decompensated period, and decompensated with structure damage, which correspond to the different stages of diabetes. Concurrently, during early diabetes, $\mathrm{qI}$ stagnation occurs due to liver depression and other syndromes. In the middle stage, intestinal dampnessheat and other syndromes occur. Finally in the middle and late stages the dual deficiency of qi and yin and the dual deficiency of yin and yang and other syndromes are present; islet $\beta$-cells are increasingly damaged. As a result, insulin secretion gradually declines as symptoms evolve. Varying degrees of insulin resistance occur with different symptoms and commonly first increase and then decline as symptoms evolve. This might be because, in the late stages of diabetes whereas the number of islet $\beta$-cells gradually decline and the number of insulin receptors on target organs relative increases.
3.2. Diabetic Patterns of Inflammatory Markers. Studies [55] have shown that CRP, IL-6, and other cytokines mediate insulin metabolic pathways, weaken insulin receptor signal transduction, induce disorders of glucose metabolism, and stimulate type 2 diabetes. Additional studies [56, 57] assessing the long-term risk factors for type 2 diabetes revealed that the levels of CRP, IL-6, IL-8, and TNF- $\alpha$ were higher in diabetic populations than the normal population. These indexes levels increase gradually as syndromes evolve; in particular, the levels of those indexes of the dual deficiency of yin and yang symptom are higher than those of any other symptoms. Consequently, IL- 6 , IL- 8 , and TNF- $\alpha$ can be used as objective indicators during the TCM-mediated syndrome differentiation of DM [58].

3.3. Diabetic Patterns of Biochemical Indicators. Fasting plasma glucose, postprandial blood sugar, and glycosylated hemoglobin were significantly higher in diabetic populations than normal individuals, but there were no significant differences among different syndromes $[59,60]$.

When combined with high uric acid (UA) hematic disease, the UA levels of type 2 diabetic patients with dual deficiency yin and yang syndrome were higher than those of other syndromes, which suggested that the dual deficiency of yin and yang might be a factor leading to elevated blood UA levels [61].

Compared with normal individuals, obvious lipid metabolic disorders occur in patients with type 2 diabetes, including increased triglycerides (TG), total cholesterol (TC), low density lipoprotein (LDL), and decreased high density lipoprotein (HDL). Phlegm and heat stasis changed the most with an increased severity of symptoms. As the course of the disease lengthened and TC and LDL-C increased, the lipid metabolism disorders of phlegm and heat stasis symptom became most serious $[62,63]$.

3.4. Diabetic Pattern Types of Hemorheology. Studies [58, 64] have shown that significant changes occur between the hemorheological performances of normal and type 2 diabetes populations. The levels of blood specific viscosity, fibrinogen, hematocrit, erythrocyte sedimentation rate (ESR), and other indicators of dual deficiency of qi and yin symptom were significantly higher than those of normal populations. This suggested that the occurrence of diabetes was associated with increased blood viscosity, and that the dual deficiency of qi and yin was the pathological basis for the change of blood rheology.

3.5. Diabetic Patterns of Related Genes. Studies [65] on the relationship between syndrome differentiation of diabetes by TCM and the level of calcitonin gene related peptide (CGRP) showed that the levels of CGRP of every syndrome of diabetes are significantly lower than normal people, and the dual deficiency of yin and yang has the lowest level of CGRP. Another study [66] to explore the relation between Chinese medicine syndrome and the gene polymorphism of 
peroxisome proliferator-activated receptor delta (PPARD)$87 \mathrm{C}>\mathrm{T}$, the genotype frequencies of $\mathrm{T} / \mathrm{C}$ and $\mathrm{C} / \mathrm{C}$ at PPARD$87 \mathrm{C}>\mathrm{T}$ are higher in the dual deficiency of yin and yang syndrome in newly diagnosed type 2 diabetes patient. Newly diagnosed type 2 diabetes patients with these alleles have higher levels of plasma glucose and lipids. This suggests that the PPARD-87C $>\mathrm{T}$ polymorphism might be a factor that affects the progression of type 2 diabetes.

An additional study [67] on the substance of dual deficiency of qi and yin from the molecular level used a diabetes gene array (Superarray Bioscience) containing 96 key diabetes-related genes to identify that 43 differentially expressed genes between normal and diabetic dual deficiency of qi and yin patients. Compared with the gene levels of normal people, 35 were upregulated and eight were downregulated. And further RT-polymerase chain reaction (RT-PCR) and Western-blotting measurement confirmed Fork head box C2 (FOXC2) and IRS-2 mRNA are specific genes of diabetic patients with dual deficiency of qi and yin syndrome.

\section{Summary}

The TCM emphasizes individualized treatment and pay attention to yin-yang balance and a holistic approach. Deep understanding of diabetes' clinical manifestation and symptom differentiation from different aspects are very important to improve the clinical effects, such as uncomfortable symptoms, blood glucose, blood lipids, and diabetes complications. Though symptom differentiation of TCM is relatively difficult to understand, with the development of modern medicine and researcher's efforts on it, people will get familiar with it and make TCM play an important role in diabetic fields.

\section{Conflict of Interests}

All authors declare that there is no conflict of interests regarding the publication of this paper.

\section{Acknowledgment}

This work was financially supported in part by the 973 Project (no. 2010CB530601).

\section{References}

[1] F. M. Lian, Z. X. Wei, X. F. Lv et al., "Clinical study on reducing sugar effect of Kaiyu Qinre-Jiangzhuo prescription on T2DM," World Journal of Integrated Traditional and Western Medicine, vol. 3, pp. 32-35, 2008.

[2] F. Lian, G. Li, X. Chen et al., "Chinese herbal medicine Tianqi reduces progression from impaired glucose tolerance to diabetes: a double-blind, randomized, placebo-controlled, multicenter trial," The Journal of Clinical Endocrinology and Metabolism, vol. 99, pp. 648-655, 2014.

[3] X. L. Tong, S. T. Wu, F. M. Lian et al., "The safety and effectiveness of TM81, a Chinese herbal medicine, in the treatment of type 2 diabetes: a randomized double-blind placebo-controlled trial," Diabetes, Obesity and Metabolism, vol. 15, no. 5, pp. 448454, 2013.
[4] W. H. Liu, Z. Q. Hei, H. Nie et al., "Berberine ameliorates renal injury in streptopzotocin-induced diabetic rats by suppression of both oxidative stress and aldose reductase," Chinese Medical Journal, vol. 121, no. 8, pp. 706-712, 2008.

[5] Y. S. Lee, W. S. Kim, K. H. Kim et al., "Berberine, a natural plant product, activates AMP-activated protein kinase with beneficial metabolic effects in diabetic and insulin-resistant states," Diabetes, vol. 55, no. 8, pp. 2256-2264, 2006.

[6] L. Y. Ma, "The clinical observation on the syndrome differentiation by TCM in the treatment of diabetes," China Healthy Industry, vol. 10, p. 135, 2013.

[7] X.-L. Tong, L. Dong, L. Chen, and Z. Zhen, “Treatment of diabetes using traditional Chinese medicine: past, present and future," The American Journal of Chinese Medicine, vol. 40, no. 5, pp. 877-886, 2012.

[8] J. X. Zhao, S. D. Wang, J. Li et al., "Studies on TCM syndrome differentiation and evaluation standards of diabetes," World Journal of Integrated Traditional and Western Medicine, vol. 8, pp. 504-506, 2013.

[9] J. P. Wei, R. Wu, and L. Lan, "Review on the study of diabetes syndromes," The Journal of Medical Research, vol. 40, pp. 7-10, 2011.

[10] X. L. Tong, W. K. Liu, J. Wang, Q. Ni, and J. P. Wei, "Key points of syndrome differentiation and practical application of stagnation, heat, deficiency and damage, four stages of diabetes," Jilin Journal of Traditional Chinese Medicine, vol. 32, pp. 442-444, 2012.

[11] H. Q. Cheng, J. Cheng, and Z. X. Wei, "The Theory study on the relationship between Qi stagnation due to liver repression and xiaoke disease," Journal of Jiangsu Traditional Chinese Medicine, vol. 18, pp. 35-36, 1997.

[12] J. P. Chen, A. C. Wang, L. Yin, and Z. F. Zhao, "Dispensable symptoms to diagnose Qi stagnation due to liver depression syndrome and its relationship with depression," Journal of Liaoning Traditional Chinese Medicine, vol. 32, pp. 19-22, 2005.

[13] G. Z. Wang, L. M. Duan, S. B. Sun, Y. J. Shen, Y. J. Chen, and H. B. Zhao, "The clinical Observation of the treatment of type 2 diabetes by coursing liver and regulating Qi," Journal Traditional Chinese Medicine, vol. 22, pp. 336-337, 2000.

[14] T. Y. Liu, "The treatment of coursing liver and regulating Qi for 63 cases of T2DM," Zhong Yi Han Shou Tong Xun, vol. 19, p. 51, 2000.

[15] X. L. Tong, Q. Ni, and J. P. Wei, "Standards of diagnosis and treatment of diabetes by TCM," World Jounal of Integrated Traditional and Western Medicine, vol. 6, pp. 540-547, 2011.

[16] X. L. Tong, G. Z. Bi, Zh. Zhen et al., "TCM syndrome differentiation of 2508 T2DM cases," World Journal of Intergrated Traditional and Western Medicine, vol. 3, pp. 26-28, 2008.

[17] W. L. Li, K. Song, and X. J. Zhang, "The application of Da Chaihu decoction in T2DM," Journal of Henan Traditional Chinese Medicine, vol. 33, pp. 336-337, 2013.

[18] X. Deng and W. J. Wang, "Da Chaihu decoction in the treatment of 39 patients with obesity diabetes," Journal of Shananxi Traditional Chinese Medicine, vol. 32, pp. 171-172, 2011.

[19] J. R. Gan, X. L. Liu, L. Li, and Y. Zhang, “The report of 1492 cases of type 2 diabetes syndrome epidemilogical survey in Kunming," Yunnan Journal of Traditional Chinese Medicine and Materia Medica, vol. 34, pp. 26-27, 2013.

[20] L. P. Zhou, J. P. Wei, F. Liu et al., "Sthdy on the TCM pathology and syndrome characteristic of first attack diabetes," Journal of Sichuan of Traditional Chinese Medicine, vol. 26, pp. 34-36, 2008. 
[21] G. F. Liu, W. K. Liu, H. Y. Ji, J. Song, and X. L. Tong, “Thought of diagnosis and treatment of T2DM by TCM," Journal of Traditional Chinese Medicine, vol. 52, pp. 1243-1245, 2011.

[22] Y. F. Gu and Q. Fu, "Study on Xiao Xianxiong decoction in the treatment of diabetes from," Phlegm, Heat and Qi Stagnation, Global Traditional Chinese Medicine, vol. 3, pp. 136-137, 2010.

[23] M. S. Jin, X. Y. Chen, and H. Y. Ji, "Analysis on dialectical points about Xiao Xianxiong Decoction in the treatment of T2DM according to Professor Tong experience," Journal of Yunnan University of Traditional Chinese Medicine, vol. 34, pp. 40-43, 2011.

[24] H. Wang and Q. Zhou, "Professor Tong's experience on Xiao Xianxiong decoction in the treatment of T2DM," Chinese Journal for Clinicians, vol. 41, pp. 68-70, 2013.

[25] H. M. Wang, M. Chen, H. Wang, and J. Xia, “TongFu Runzao decoction in the treatment of oral hypoglycemic drugs in uncontrolled stomach-intestine excessive heat type 2 diabetes 60 cases," Chinese Journal of Experiment Traditonal Chinese Medicine Formula, vol. 19, pp. 273-276, 2013.

[26] X. L. Tong, "Application of a series of classical prescriptions with rhizoma coptidis being monarch drug in the syndrome differentiation of diabetes," Journal of Traditional Chinese Medicine, vol. 54, pp. 209-221, 2013.

[27] X. Sun, "Application of Xie Xin decoction and likewise decoctions in the treatment of diabetes," Journal of Traditional Chinese Medicine, vol. 51, pp. 114-116, 2010.

[28] L. H. Zhao, H. Y. Ji, B. W. Ji, J. Song, and X. L. Tong, "Exploration of Gegen Qinlian decoction's effect on diabetes mellitus in theory," Chinese Journal of Traditional Chinese Medicine and Pharmacy, vol. 27, pp. 280-283, 2012.

[29] N. P. Huang, "Clinicial effects of Gegen Qinlian decoction combined with metfom infor treatment on patients with type 2 diabetes of dampness heat syndrome, seek medical and aslc," The Medicine, vol. 10, pp. 229-300, 2012.

[30] S. M. Li, Y. C. Li, W. G. Li, and Z. G. Wang, "Investigtion on characteristics of diabetic Dampness Heat Syndrome and evolving regulation," Journal of Hunan University of Traditional Chinese Medicine, vol. 27, pp. 65-68, 2007.

[31] Y. H. Wang, "The TCM treatment of dampness heat syndrome of diabetes," Journal of Fujian College of TCM, vol. 13, pp. 32-33, 2003.

[32] J. R. Gan and Y. Q. Chen, "Study on syndrome differentiation of TCM," Journal of Yunnan University of Traditional Chinese Medicine, vol. 35, pp. 41-45, 2012.

[33] Q. M. Zhang, Z. Q. Chen, Y. Z. Liu et al., "A survey on the pattern differentiation of type 2 diabetic mellitus and its complications," Journal of TCM University of Hunan, vol. 24, pp. 33-36, 2004.

[34] X. Y. Zhou, "A part of Li Zhuo's experience about Baihu Renshen decoction in the treatment of Xiaoke disease," Journal of Practical Traditional Chinese Internal Medicine, vol. 17, p. 161, 2003.

[35] L. Xin, F. S. Fu, L. K. Liao, and L. Q. Deng, "Clinical research of Xiaoke Fang in the tretment of oral glucose-lowering drugs controlled type 2 diabetes," China Journal of Chinese Medicine, vol. 28, pp. 1215-1217, 2013.

[36] H. Liu, K. K. Wei, J. P. Li, and S. W. Ma, "Xiaoke Fang in the treatment of diabetes 200 cases," Journal of TCM of Shanxi, vol. 26, pp. 1322-1323, 2005.

[37] L. Lin and Q. Ni, Theory of Practice of III-Type Differentiation of T2DM, Scitific Chinese, 2011.
[38] C. X. Xu, W. C. Ye, and Y. G. Hu, "Syndome differentiation and correlative analysis of 120 cases of type 2 diabetes mellius," Shanghai Journal of Traditional Chinese Medicine, vol. 41, pp. 34-36, 2007.

[39] Z. J. Mao, Y. Dong, J. Luan et al., "Investigation of TCM symptoms and syndrome in 180 patients with T2DM," Chinese Journal of Traditional Chinese Medicine and Pharmacy, vol. 24, pp. 1064-1067, 2009.

[40] Z. Li, S. D. Wang, T. Tan et al., "Exploration on distribution regulation of TCM syndromes in 2735 cases of type 2 diabetes and pathogenesis of vigorous fire consuming Qi," World Chinese Medicine, vol. 8, pp. 488-490, 2013.

[41] X. C. Tang, "Jiawei Shengmai decoction in the treatment of 60 patients with T2DM," Henan Traditional Chinese Midecine, vol. 27, pp. 52-53, 2007.

[42] P. Wang and Y. Wang, "Application of Shengmai decoction in the treatment of diabetes," Journal of Tianjin University of Traditional Chinese Medicine, vol. 30, pp. 127-128, 2011.

[43] B. Dai and Z. Y. Ou, "Study on Qiju Dihuang decoction in the treatment of insufficiency of the liver and kidney syndrome of the old with diabetes," Clinical Journal of Traditional Chinese Medicine, vol. 17, pp. 544-545, 2005.

[44] W. T. Zhang, "Qiju Dihuang decoction in the treatment of diabetic retinopathy in 36 cases," Chinese Community Doctors, vol. 15, p. 68, 2013.

[45] B. L. He, Y. P. Tang, G. B. Zhuang, and J. N. Chen, "Observation of the nearly and long-term clinical effects of Qiju Dihuang decoction in the treatment of diabetic retinopathy," China Medical Herald, vol. 6, pp. 83-118, 2009.

[46] Y. E. Lu, "Jingui Shenqi decoction combined with western drugs in the treatment of DN in 60 cases," Journal of Shaanxi College of Traditonal Chinese Medicine, vol. 32, pp. 29-31, 2009.

[47] X. M. Yang, "Jingui Shenqi decoction in the treatment of T2DM in 120 cases," Chinese Journal of Experimental Traditional Medicine Formula, vol. 17, pp. 261-263, 2011.

[48] H. Z. Wu, "Clinical observation of Jingui Shenqi decoction in the treatment of T2DM," Pharmacology and Clinics of Chinese Materia, vol. 29, pp. 191-193, 2013.

[49] D. H. Liu, "Observation of Jingui Shenqi Decoction in the treatment of 62 patients with Dual Deficiency of Yin and Yang Syndrome of T2DM," Journal of New Chinese Medicine, vol. 36, pp. 31-32, 2004.

[50] G. M. Gong, L. G. Yuan, J. Li et al., "Epidemiology of chronic diabetic complications and research of syptoms of traditional chinese medicine of newly-diagnosed type 2 diabetes," Journal of Liaoning Traditional Chinese Medicine, vol. 39, pp. 26-30, 2012.

[51] X. L. Tong, Y. Zhao, G. Z. Bi et al., "Research on application of preventive "Treatment of Disease" and "Collateral Disease" theories in diabetic microvascular complications," Journal of Traditional Chinese Medicine, vol. 48, pp. 485-486, 2007.

[52] Y. Zhao, X. L. Tong, and L. Chen, "Relationship between the Function evolving of $\beta$-cell and TCM syndrome differentiation of diabetes," Journal of Shandong Traditional Chinese Medicine, vol. 25 , pp. 3-5, 2006.

[53] J. Q. Zhang, K. Ma, and S. G. Lv, "Relationship between TCM syndrom differentiation and insulin resistance of type 2 diabetes," China of Shandong Traditional Chinese Medicine, vol. 25, p. 3, 2006.

[54] Z. Y. Gao, S. P. Qian, X. M. Yi, X. P. Luo, and X. D. Huang, "Relationship between TCM syndrome differentiation and the 
function of $\beta$-cell and Insulin sensitivity," Chinese Journal of Integrative Medicine on Cerebravasoular Disease, vol. 1, p. 58 , 2003.

[55] S. Wassmann, M. Stumpf, K. Strehlow et al., "Interleukin6 induces oxidative stress and endothehal dysfunction by overexpression of the angiotensin ii type 1 receptor," Circulation Research, vol. 94, no. 4, pp. 534-541, 2004.

[56] F. B. Hu, J. B. Meigs, T. Y. Li, N. Rifai, and J. E. Manson, "Inflammatory markers and risk of developing type 2 diabetes in women," Diabetes, vol. 53, no. 3, pp. 693-700, 2004.

[57] R. Bouhaha, T. Baroudi, H. Ennafaa et al., "Study of TNF $\alpha$ 308G/A and IL6 -174G/C polymorphisms in type 2 diabetes and obesity risk in the Tunisian population," Clinical Biochemistry, vol. 43, no. 6, pp. 549-552, 2010.

[58] J. Y. Xu, Z. Q. Chen, N. Y. Hu, and C. H. Chen, "Investigation of the relationship between the syndrome patterns in TCM differentiation diagnostics and the levels of plasmatic IL-6, IL8 and TNF-a in diabetes mellitus patients," China Journal of Modern Medicine, vol. 13, pp. 66-67, 2003.

[59] L. Y. Zhao, L. F. Bi, H. H. Zhao et al., "Correlation between syndrome types and clinical indexes in 147 patients with type 2 diabetes," Journal of Beijing University of Traditional Chinese Medicine, vol. 36, pp. 480-483, 2013.

[60] H. Li and J. Peng, "Study on relationship between TCM syndrome differentiation of T2DM and dyslipidemia, blood glucose and body mass index," Chinese Journal of Basic Medicine in Traditional Chinese Medicine, vol. 11, pp. 925-926, 2005.

[61] Y. Gong and H. Wang, "Distribution of blood uric acid level in dual deficiency Qi and Yin of T2DM," Xinjiang Journal of Traditional Chinese Medicine, vol. 25, pp. 15-16, 2007.

[62] X. Wang, L. Zhao, and X. R. Xu, "Study on correlation among stagnated heat syndrome of type 2 dibetes liquid metabolic and disease course," Journal of Liaoning University of TCM, vol. 15, pp. 5-6, 2013.

[63] L. B. Zhou, M. Li, L. Dong, and X. L. Tong, "The clinical study of the relationship between T2DM syndrome differentiation by TCM of young obese patients and lipid metabolism," Jiangsu Journal of Traditional Chinese Medicine, vol. 40, pp. 43-45, 2008.

[64] L. X. Zhong and Y. Q. Tu, "Change of blood rheology markers in II type diabetes patients," Zhejiang Medical Education, vol. 4, pp. 41-43, 2005.

[65] Z. Y. Li and L. Q. Zhang, "Study on TCM syndrome differentiation of T2DM, plasma endothelin and calcitonin gene related peptode," Journal of Fujian College of Traditional Chinese Medicine, vol. 11, pp. 1-4, 2001.

[66] Ch. Ren, F. T. Geng, and J. W. Qian, "Relation between Chinese medicine Syndrome and PPARD-87C $>\mathrm{T}$ gene polymorphism in newly diagnosed type 2 diabetes patients," Journal of Traditional Chinese Medicine, vol. 53, pp. 1397-1400, 2012.

[67] K. F. Chai, X. L. Huang, and J. W. Qian, "Gene expression study of type 2 diabetes mellitus belong to defficiency of both Qi and Yin," Chinese Archives of Traditional Chinese Medicine, vol. 27, pp. 1351-1354, 2009. 


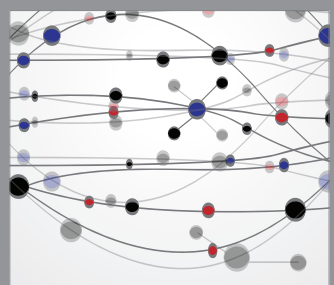

The Scientific World Journal
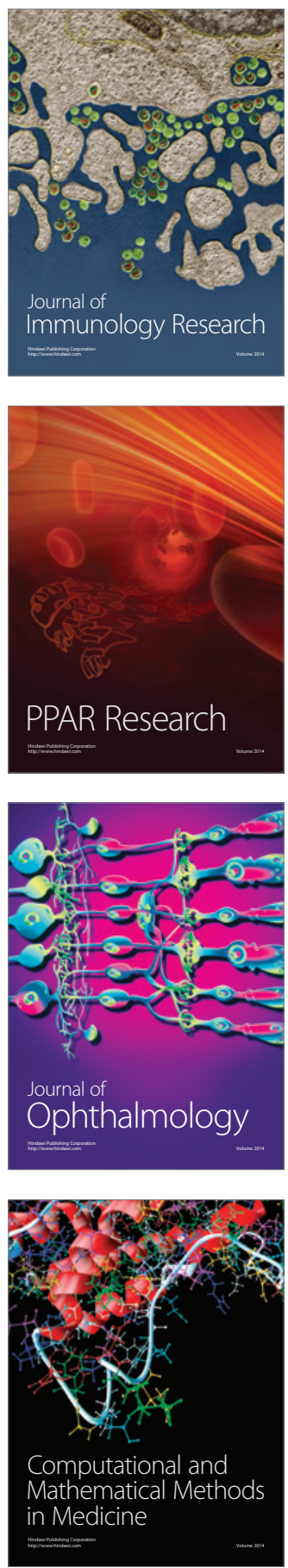

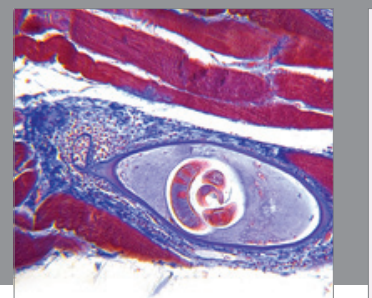

Gastroenterology

Research and Practice
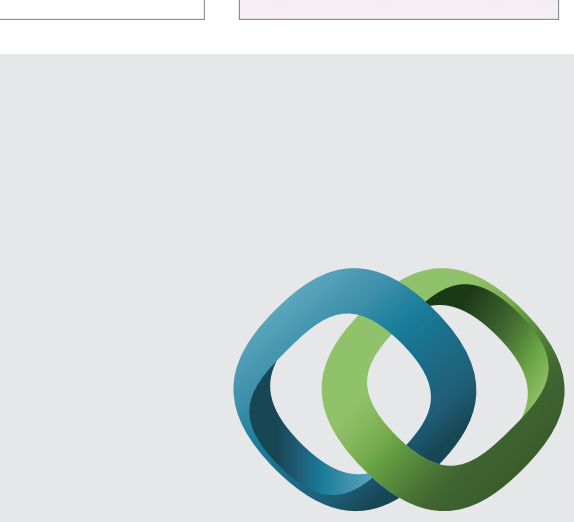

\section{Hindawi}

Submit your manuscripts at

http://www.hindawi.com
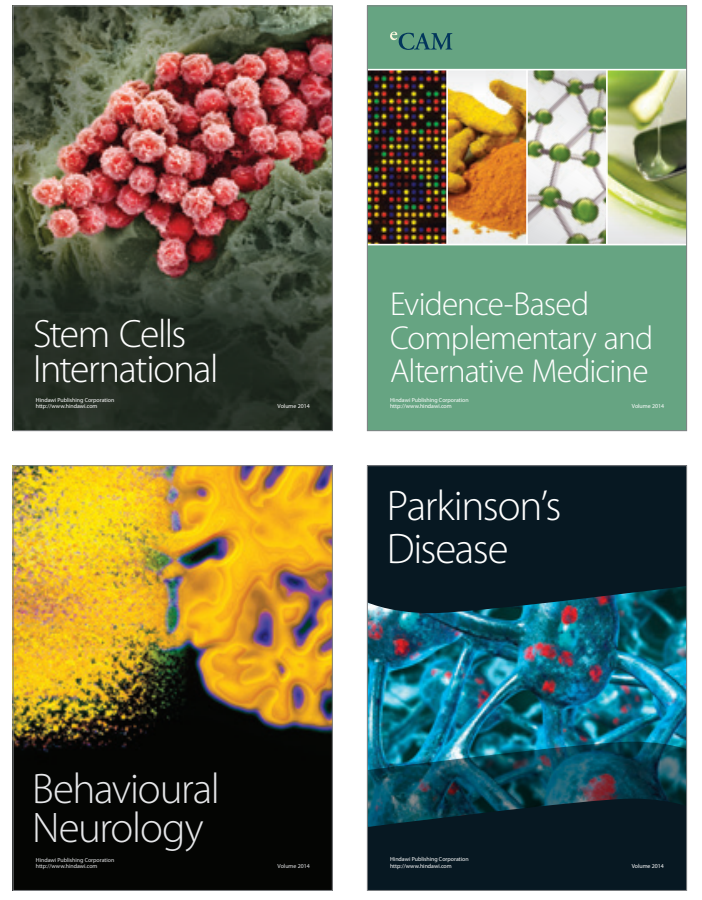
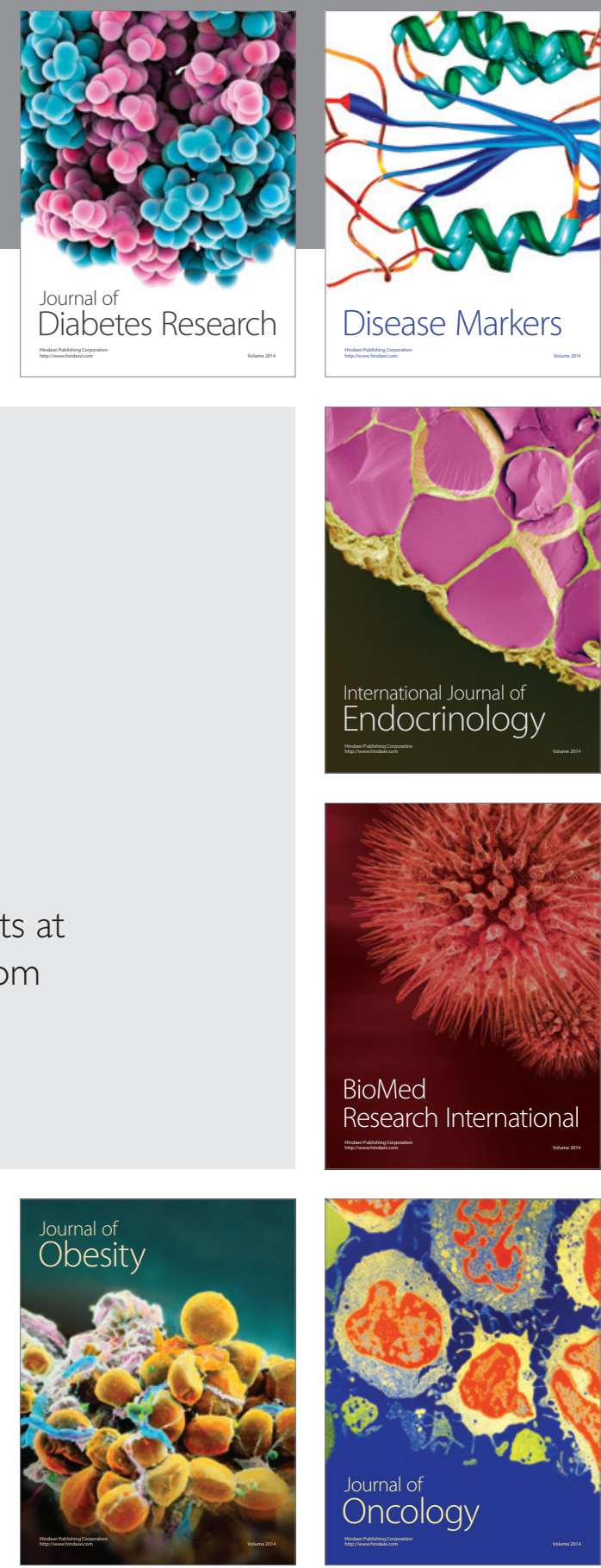

Disease Markers
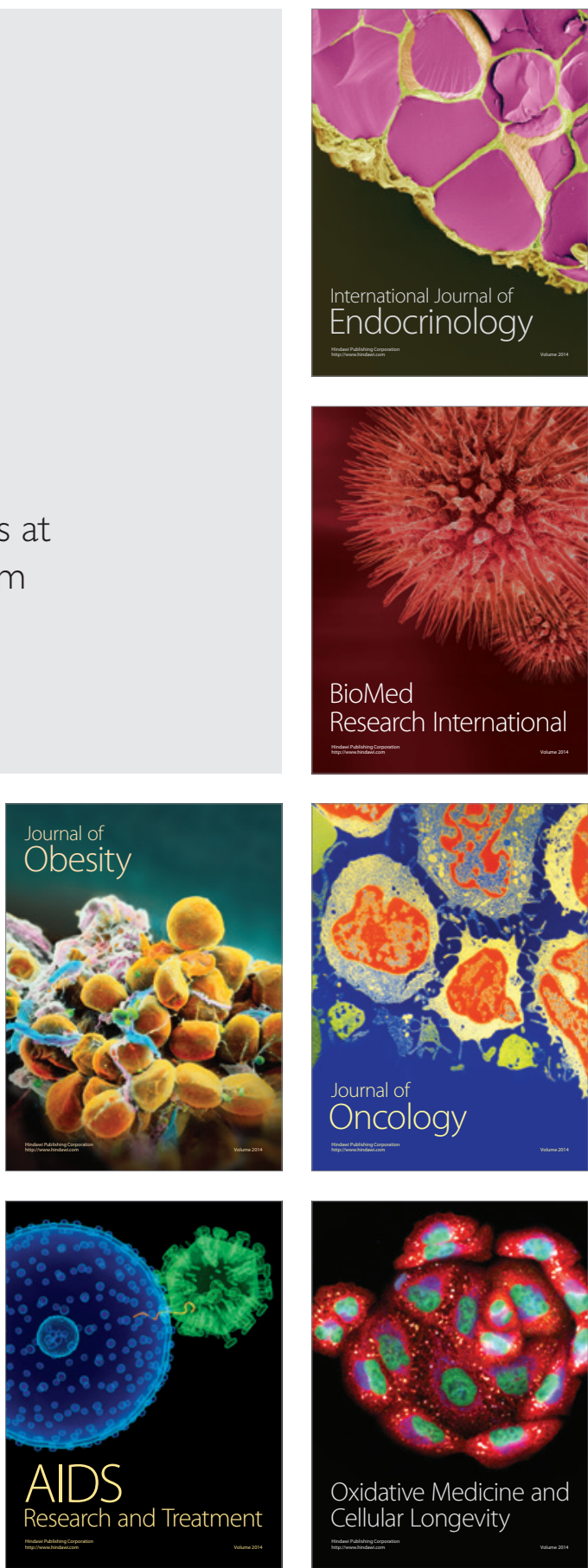